\title{
FAKE $R^{4}$ 's, EINSTEIN SPACES AND SEIBERG-WITTEN MONOPOLE EQUATIONS
}

\author{
Cihan Saçlıoğlu ${ }^{1,2}$ \\ ${ }^{1}$ Physics Department, Bog̃aziçi University \\ 80815 Bebek-İstanbul, Turkey \\ and \\ ${ }^{2}$ Feza Gürsey Institute \\ Bog̃aziçi University-TUBITAK \\ 81220 Çengelköy, Istanbul- Turkey
}

\begin{abstract}
We discuss the possible relevance of some recent mathematical results and techniques on four-manifolds to physics. We first suggest that the existence of uncountably many $R^{4}$ 's with non-equivalent smooth structures, a mathematical phenomenon unique to four dimensions, may be responsible for the observed four-dimensionality of spacetime. We then point out the remarkable fact that self-dual gauge fields and Weyl spinors can live on a manifold of Euclidean signature without affecting the metric. As a specific example, we consider solutions of the Seiberg-Witten Monopole Equations in which the $U(1)$ fields are covariantly constant, the monopole Weyl spinor has only a single constant component, and the 4-manifold $\mathcal{M}_{4}$ is a product of two Riemann surfaces $\Sigma_{p_{1}}$ and $\Sigma_{p_{2}}$. There are $p_{1}-1\left(p_{2}-1\right)$ magnetic(electric) vortices on $\Sigma_{p_{1}}\left(\Sigma_{p_{2}}\right)$, with $p_{1}+p_{2} \geq 2\left(p_{1}=p_{2}=1\right.$ being excluded). When the two genuses are equal, the electromagnetic fields are self-dual and one obtains the Einstein space $\Sigma_{p} \times \Sigma_{p}$, the monopole condensate serving as the cosmological constant.
\end{abstract}




\section{Introduction:}

Some time after the discovery of uncountably many four-manifolds that are homeomorphic but not diffeomorphic to $R^{4}$ [1], [2], Atiyah [3] challenged physicists to find a physical realization for these 'Fake $R^{4}$ 's'. Our first aim is to suggest a possible response to this challenge. We will argue in the next section that spacetime may be four-dimensional because of this uncountable profusion of Fake $R^{4}$ 's, as opposed to only a single standard $R^{n}$ in any other dimension $n$.

In the rest of the note we examine the relationship between the SeibergWitten monopole equations (SWME) [4] and physics. The physics interest in solutions of Euclidean matter-coupled gravity has to do with semiclassical treatments of vacuum tunnelling, estimating the path integral of gravity and possibly for understanding the earliest phases of the universe. Some mathematical questions one may raise about such manifolds are: (i) which smooth compact manifolds admit Einstein metrics, and, (ii) how many (modulo diffeomorphisms and rescalings) different Einstein metrics can be put on the same manifold [5]. In ref. [5] global methods are employed. Our approach, in contrast, is based on explicit local equations and their solutions; these provide a pedestrian route to Einstein spaces and $\operatorname{Spin}_{c}$ structures. Since most of these results have already been presented elsewhere [6], the discussion will be rather condensed.

\section{Fake $R^{4}$ 's and spacetime:}

The idea that the dimensionality of spacetime can be derived from some dynamical principle rather than being accepted as an a priori fact is a feature of String theory, so it may be instructive to examine how this is handled in the Polyakov path integral formulation of strings. The measure consists of $\left[d X^{\mu}\right]\left[g_{a b}\right]$, but it is customary to treat target space coordinates and the world sheet very differently. For the latter, care is taken to include (i) all possible world sheet topologies (by performing a sum over genera), (ii) all inequivalent complex structures (by integrating over moduli space). On the other hand, for no obvious or compelling reason, similar considerations are not extended to target space, a.k.a. the spacetime manifold. A critical upper limit for the dimension of spacetime emerges indirectly when one demands cancellation between the ghost and target space contributions to the anomaly, but this is clearly different from the deliberate choices one allows for the world 
sheet. One then appeals to the possibility of space being curved in General Relativity and argues that some as yet not understood physical mechanism closes all but three space dimensions onto themselves.

Here we would like to propose tentatively another possible physical mechanism leading to a four-dimensional spacetime. Basically, the idea is to let a generalized path integral decide the dimensionality of the target spacetime. A path integral for quantizing Einstein's theory involving a sum over various topologies has already been considered [7]. In similar fashion, imagine extending the String theory path integral by allowing a sum over all possible target space manifolds. In partial analogy with what is done with the world sheet, the sum could be performed as follows: Choose a particular dimension $D$, sum over all possible topologies at that dimension just as one sums over the sphere, the torus, etc. We note at this point that the physical signatures of different global 'Cosmic Topologies' for four-dimensional spacetime have already been examined [8]. Next, when the dimension admits them, sum over inequivalent differentiable structures. When finished with that particular dimension, move on to $D+1$. Admittedly, the sum cannot be made precise without knowledge, among other things, of the Boltzmann factors to be assigned to the different differentiable structures. However, a very gross fact at $D=4$ renders this knowledge superfluous if the Boltzmann factors of these structures are comparable. The uncountable number of $R^{4}$ 's, all but one of which are "fake", completely overwhelms all other contributions from other dimensions and topologies. Since fake and standard $R^{4}$ 's cannot be distinguished by looking at a local neighborhood, but only by comparing the overall global structure [9], the argument would suggest that the familiar local $R^{4}$ we live in is in fact some average of the standard one and all its fakes. The differentiable structure being independent of the metric, the above considerations hold regardless of the signature of the $R^{4}$ metric.

\section{Einstein spaces and Seiberg-Witten monopole equations:}

We start by some very general facts, which, however, lead to surprising conclusions about physics on Euclidean manifolds. Recall that as we go from $(-+++)$ signature to $(++++)$, the Dirac bilinear covariants $\psi^{\dagger} \gamma^{0} \Gamma \psi$ turn into $\psi^{\dagger} \Gamma \psi$. In a Weyl representation, the $\gamma^{\mu}$ are block-off diagonal, while $\gamma^{5}$ is block diagonal; they are all Hermitean. We may take, for example, $\gamma^{i}=\tau_{1} \otimes \sigma_{i}, \gamma^{4}=\tau_{2} \otimes I$ and $\gamma^{5}=\tau_{3} \otimes I$. This means that in Euclidean 
signature the vector and the axial vector currents and the energy-momentum tensor for a Weyl spinor automatically vanish! Now these are precisely the bilinears which would have served as sources for the gauge field strength tensor, its dual, and the Einstein tensor, respectively. Hence the gauge fields and the metric are unaffected by the Weyl fields, and the field strengths are covariantly constant.

Euclidean signature also allows real, non-trivial self-dual or anti-self-dual gauge fields. These automatically solve the sourceless Maxwell's equations. Furthermore, the energy-momentum tensor for these fields also vanishes, and we have the vacuum Einstein field equations

$$
\mathcal{R}_{\mu \nu}-\frac{1}{2} g_{\mu \nu} \mathcal{R}=\Lambda g_{\mu \nu}
$$

where we have allowed a cosmological constant $\Lambda$. This is just the defining equation for the Einstein spaces considered in [5]. What we have said so far holds for all gauge fields, whether Abelian or not, and also for Weyl spinors which are not necessarily singlets under the gauge group. However, from now on, we will work with a $U(1)$ gauge field $A_{\mu}$ and a single Weyl spinor $\psi$, which then satisfy the Dirac equation

$$
\not D_{A} \psi=\gamma^{a} E_{a}^{\mu}\left(\partial_{\mu}+i A_{\mu}+\frac{1}{8} \omega_{\mu}^{b c}\left[\gamma_{b}, \gamma_{c}\right]\right) \psi=0
$$

where $\omega_{\mu}^{b c}$ are the spin connection coefficients and $E_{a}^{\mu}$ the inverses of the vierbeins.

If one assumes that the Weyl spinor represents a massless monopole, the above are exactly the fields in the SWME. The SWME have proven to be a considerably more practical tool for distinguishing inequivalent smooth structures on homeomorphic four-manifolds than the earlier Donaldson theory (the recent realization that [10], [1] Seiberg-Witten-Donaldson theory still fails to detect certain inequivalent smooth structures does not affect the present analysis). The SWME consist of (2) and

$$
F_{\mu \nu}^{+} \equiv \frac{1}{2}\left(F_{\mu \nu}+\frac{1}{2} \epsilon_{\mu \nu \alpha \beta} F^{\mu \nu}\right)=-\frac{i}{4} \psi^{\dagger}\left[\gamma_{\mu}, \gamma_{\nu}\right] \psi
$$

The SWME have at least one solution $\left(A_{\mu}, \psi\right)$ for every metric $g_{\mu \nu}$ on the manifold [5]. This is related to the mathematical fact that even when a 4manifold does not admit a spin structure because of an obstruction in the 
form of a spinor phase ambiguity, the introduction of a $U(1)$ bundle can compensate for the phase mismatch. One then has a Spin structure [12] $^{-12}$ and spinors are allowed [13]. Using the Weitzenbock-Lichnerowicz formula, one can further show that (2) and (3) do not admit non-trivial non-singular solutions unless the scalar curvature $\mathcal{R}$ is negative somewhere [14]. If one now tries to find simultaneous solutions of (11), (2), (3) and $F_{\mu \nu}=\widetilde{F_{\mu \nu}}$, it is clear that only constant negative curvature Einstein spaces will admit nonsingular matter fields. Putting these facts together, we see that all possible simultaneous non-singular, non-trivial solutions of the SWME and (1) require self-dual gauge fields and constant negative curvature. We will give explicit examples of these in the later sections. Semi-trivial solutions without spinors and anti-self-dual fields are also a possibility, as are non-trivial but singular solutions with self-dual gauge fields.

4. Explicit solutions of the form $\mathcal{M}_{2}^{(1)} \times \mathcal{M}_{2}^{(2)}$ :

As an example which permits simple explicit calculations, we look for solutions of the SWME in the form of a product of two two-manifolds parametrized by coordinates $\left(x^{1}, x^{2}\right)$ and $\left(x^{3}, x^{4}\right)$; it is then natural to assume that in $\left(A^{1}, A^{2}, A^{3}, A^{4}\right)$ the first two components depend on the first pair of coordinates and the last two on the second pair. The SWME then imply that $\psi$ has only one non-vanishing component, say $\psi_{1}$, which is forced to be a constant. The scalar curvature $R=2 R_{12}^{12}+2 R_{34}^{34}$ turns out to have the value $-2\left|\psi_{1}\right|^{2}$, so the solutions are guaranteed to be non-singular. Introducing another constant $|\phi|$, we can break up $R$ into the curvatures of $\mathcal{M}_{2}^{(1)}$ and $\mathcal{M}_{2}^{(2)}$ by writing $R_{12}^{12}=-|\phi|^{2}$ and $R_{34}^{34}=-\left(\left|\psi_{1}\right|^{2}-|\phi|^{2}\right)$. We then see that there are three kinds of solutions in which (i) both manifolds have constant negative curvature $\left(\left|\psi_{1}\right|>|\phi|\right)$, (ii) one is flat and the other has constant negative curvature $\left(\left|\psi_{1}\right|=|\phi|\right)$ or $|\phi|=0$ ), and (iii) one has positive and the other constant negative curvature $\left(\left|\psi_{1}\right|<|\phi|\right)$, but the total scalar curvature is still negative. In terms of dimensionless complex coordinates $z^{1} \equiv \sqrt{2}|\phi|\left(x^{1}+i x^{2}\right)$ and $z^{2} \equiv \sqrt{2}\left(\left|\psi_{1}\right|^{2}-|\phi|^{2}\right)^{\frac{1}{2}}\left(x^{3}+i x^{4}\right)$, the solution for case (i) is

$$
\begin{aligned}
& \omega_{2}^{1}=-i\left\{\frac{1}{2} d \ln \left(\frac{d \bar{g}_{1}}{d \bar{z}_{1}} \frac{d z_{1}}{d g_{1}}\right)+\frac{\left(g_{1} d \bar{g}_{1}-\bar{g}_{1} d g_{1}\right)}{\left(1-g_{1} \bar{g}_{1}\right)}\right\}, \\
& \omega_{4}^{3}=-i\left\{\frac{1}{2} d \ln \left(\frac{d \bar{g}_{2}}{d \bar{z}_{2}} \frac{d z_{2}}{d g_{2}}\right)+\frac{\left(g_{2} d \bar{g}_{2}-\bar{g}_{2} d g_{2}\right)}{\left(1-g_{2} \bar{g}_{2}\right)}\right\},
\end{aligned}
$$




$$
\begin{gathered}
A=-\frac{1}{2}\left(\omega_{2}^{1}+\omega_{4}^{3}\right), \\
R_{2}^{1}=-2 i \frac{d g_{1} \wedge d \bar{g}_{1}}{\left(1-g_{1} \bar{g}_{1}\right)^{2}}, \quad R_{4}^{3}=-2 i \frac{d g_{2} \wedge d \bar{g}_{2}}{\left(1-g_{2} \bar{g}_{2}\right)^{2}}, \\
F=i \frac{d g_{1} \wedge d \bar{g}_{1}}{\left(1-g_{1} \bar{g}_{1}\right)^{2}}+i \frac{d g_{2} \wedge d \bar{g}_{2}}{\left(1-g_{2} \bar{g}_{2}\right)^{2}}=-\frac{1}{2}\left(R_{2}^{1}+R_{4}^{3}\right), \\
d s^{2}\left(\mathcal{M}_{2}^{(1)}\right)=\frac{d g_{1} d \overline{g_{1}}}{\left(1-g_{1} \overline{g_{1}}\right)^{2}}, \\
d s^{2}\left(\mathcal{M}_{2}^{(2)}\right)=\frac{d g_{2} d \overline{g_{2}}}{\left(1-g_{2} \overline{g_{2}}\right)^{2}},
\end{gathered}
$$

where $g_{1}\left(z_{1}\right)$ and $g_{2}\left(z_{2}\right)$ are arbitrary analytic functions originating from Liouville equations, which in turn were obtained from the SWME using our Ansatz.

We will illustrate case (ii) by taking $|\phi|=0$, which corresponds to a flat metric for the first manifold, and one of constant negative curvature for the second. The solutions for the first manifold are then no longer characterized by a solution of the Liouville equation, but instead by harmonic functions, which we will later take as the real part of an analytic function $h\left(z_{1}\right)$. The results then follow from (4)-(10) by setting $g_{1}=0$ except in (9), where the metric is now flat; note also the first two components of the $U(1)$ connection are absent.

In the final case (iii), we take the second manifold as the one with positive curvature and the solution is given by

$$
\begin{gathered}
\omega_{2}^{1}=-i\left\{\frac{1}{2} d \ln \left(\frac{d \bar{g}_{1}}{d \bar{z}_{1}} \frac{d z_{1}}{d g_{1}}\right)+\frac{\left(g_{1} d \bar{g}_{1}-\bar{g}_{1} d g_{1}\right)}{\left(1-g_{1} \bar{g}_{1}\right)}\right\}, \\
\omega_{4}^{3}=i\left\{\frac{1}{2} d \ln \left(\frac{d \bar{g}_{2}}{d \bar{z}_{2}} \frac{d z_{2}}{d g_{2}}\right)+\frac{\left(g_{2} d \bar{g}_{2}-\bar{g}_{2} d g_{2}\right)}{\left(1+g_{2} \bar{g}_{2}\right)}\right\}, \\
A=-\frac{1}{2}\left(\omega_{2}^{1}+\omega_{4}^{3}\right), \\
R_{2}^{1}=-2 i \frac{d g_{1} \wedge d \bar{g}_{1}}{\left(1-g_{1} \bar{g}_{1}\right)^{2}}, \quad R_{4}^{3}=+2 i \frac{d g_{2} \wedge d \bar{g}_{2}}{\left(1+g_{2} \bar{g}_{2}\right)^{2}}, \\
F=i \frac{d g_{1} \wedge d \bar{g}_{1}}{\left(1-g_{1} \bar{g}_{1}\right)^{2}}-i \frac{d g_{2} \wedge d \bar{g}_{2}}{\left(1+g_{2} \bar{g}_{2}\right)^{2}}=-\frac{1}{2}\left(R_{2}^{1}+R_{4}^{3}\right),
\end{gathered}
$$




$$
d s^{2}\left(\mathcal{M}_{2}^{(2)}\right)=\frac{d g_{2} d \overline{g_{2}}}{\left(1+g_{2} \overline{g_{2}}\right)^{2}}
$$

The equations (14)-(16) show that points on the manifold are projected stereographically onto the $g_{1}$ and $g_{2}$ complex planes. The topological properties of the manifolds are encoded in the functions $g_{1}, g_{2}$ and $h$. The simplest cases are $g_{1}=z_{1}, g_{2}=z_{2}$, giving a product of two hyperboloids in case (i), and a hyperboloid and a sphere in case (iii). Similarly, in case (ii), the simplest version of the flat metric $d s^{2}=\left|\partial h\left(z_{1}\right)\right|^{2} d z_{1} \overline{d z_{1}}$ is for $h\left(z_{1}\right)=z_{1}$; the second manifold is again a hyperboloid. However, if we choose $h$ to be an inverse elliptic function, $\mathcal{M}_{2}^{(1)}$ is turned into $T^{2}$. Similarly, the hyperboloid with coordinates $g_{1}\left(z_{1}\right)$ becomes the Riemann surface $\Sigma_{p_{1}}$ of genus $p_{1}$ when $g_{1}\left(z_{1}\right)$ is the Fuchsian function [15] (used in uniformizing an algebraic function defined on the same Riemann surface) because the Fuchsian function tessellates the hyperboloid into $4 p_{1}$-gons with geodesic edges identified in the standard way 16]. Finally, in case (iii), $\mathcal{M}_{2}^{(2)}=S^{2}$, and no such topologically different variants are possible. In this case, the most general one-to-one mapping of the Riemann sphere to itself is of the form

$$
g_{2}\left(z_{2}\right)=\frac{a z_{2}+b}{c z_{2}+d}
$$

Summarizing, we have found explicit solutions of the SWME in the form $\Sigma_{p_{1}} \times \Sigma_{p_{2}}$, with the constraint that $p_{1}+p_{2} \geq 2$, excluding $p_{1}=p_{2}=1$. Physically, $p_{1}-1\left(p_{2}-1\right)$ is the number of magnetic(electric) vortices on $\mathcal{M}_{2}^{(1)}\left(\mathcal{M}_{2}^{(2)}\right)$.

Are these solutions unique? This can be partially answered by computing the virtual dimension $W$ of the moduli space of solutions defined by $-\frac{(2 \chi+3 \sigma)}{4}+c_{1}^{2}$. For our solutions, it is easy to show that the signature $\sigma=0$, the Euler characteristic $\chi=4(p-1-1)\left(p_{2}-1\right)=2 c_{1}^{2}$; hence $W=0$. The solutions thus correspond to a discrete set of points in moduli space.

5. Einstein spaces of the form $\Sigma_{p} \times \Sigma_{p}, p \geq 2$ :

Of the solutions so far displayed, the ones with covariantly constant selfdual $U(1)$ fields are guaranteed also to solve the coupled Einstein-MaxwellDirac equations by the discussion in section 3. A glance at these solutions shows that the $U(1)$ connection over each manifold is just minus one-half of the spin connection for the same manifold; self duality thus makes the two manifolds identical, with $p-1$ magnetic vortices on the first and the 
same number of electric vortices on the other. The total scalar curvature $R=-2\left|\psi_{1}\right|^{2}$ by the SWME and this shows that the cosmological constant $\Lambda$ consists of the constant massless magnetic monopole condensate. These solutions may be regarded as an extension of the Bertotti-Robinson [17, [18] solutions, with Weyl spinors added, to Euclidean signature. Explicit computation, both analytical, and by using REDUCE, shows that none of the other SWME solves the Einstein-Maxwell-Dirac equations.

What would have happened had we chosen $\left|\psi_{1}\right|=0,\left|\psi_{2}\right| \neq 0$ in the beginning? Examining the formulae, it is easy to see that this replaces complex variables with their complex conjugates, changes the sign of the $U(1)$ fields but leaves the metric unchanged. Recalling that the two components of a Weyl spinor correspond to particles and antiparticles of the same handedness, we see that the charge conjugate of the original solution is obtained.

\section{Acknowledgements}

I am grateful to S. Akbulut for private instruction in Seiberg-Witten theory, to P. Argyres for clarifying a point involving the virtual dimension, and to Y. Nutku for informing me of [17] and [18], as well as for the REDUCE check. I thank R. P. Langlands for observing that fake and standard $R^{4}$ 's are locally indistinguishable, M. Arık, T. Dereli and R. Güven for useful discussions, and U. Kayserilioglu for help with LATEX.

\section{References}

[1] Gompf R 1983 J. Diff. Geo. 18317

[2] Taubes C H 1982 J. Diff. Geo. 17139

[3] Atiyah M 1988 Hermann Weyl Symposium Lecture in Collected Works of Michael Atiyah V 5 Oxford University Press

[4] Witten E 1994 Math. Res. Lett. 1769

[5] LeBrun C 1998 in "The Geometric Universe", ed. by S. A. Huggett, L. J. Mason, K. P. Tod, S. T. Tsou and N. M. J. Woodhouse, Oxford p.109

[6] Saclioglu C 2000 Class. Quantum Grav. 17485 
[7] Gibbons G W, Hawking S W and Perry M J 1978 Nucl. Phys. B138 141

[8] Lachièze-Rey M and Luminet J-P 1995 Phys. Rep. 254135

[9] Freed D S and Uhlenbeck K K 1991 Instantons and Four-Manifolds, Second Edition Springer-Verlag

[10] Wang S 1995 Math. Res. Lett. 2305

[11] Fintushel R and Stern R J 1998, math.SG/9811019.

[12] Eguchi T, Gilkey P B and Hanson A J 1980 Phys. Rep. 66369

[13] Morgan J W 1996 The Seiberg-Witten Equations and Applications to the Topology of Smooth Four-Manifolds Princeton University Press Mathematical Notes 44 Princeton New Jersey

[14] Akbulut S 1996 Tr. J. of Mathematics 20 (Proc. of the 4th Gökova Geometry-Topology Conference) 95

[15] Nehari Z 1952 Conformal Mapping Dover, NY

[16] Dubrovin B A, Fomenko T A and Novikov S P 1985 Modern Geometry Vol. 2, Springer-Verlag, NY

[17] Bertotti B 1959 Phys. Rev. 1161331

[18] Robinson I 1959 Bull. Acad. Pol. Sci. 7351 